\title{
Almost equitable partitions and new necessary conditions for network controllability *
}

\author{
Cesar O. Aguilar and Bahman Gharesifard

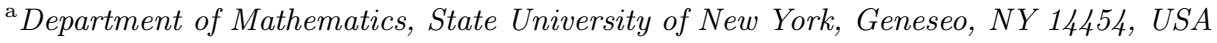 \\ ${ }^{\mathrm{b}}$ Department of Mathematics and Statistics, Queen's University, Kingston, ON KrL 3N6, Canada
}

\begin{abstract}
In this paper, we consider the controllability problem for multi-agent networked control systems. The main results of the paper are new graph-theoretic necessary conditions for controllability involving almost equitable graph vertex partitions. We generalize the known results on the role of graph symmetries and uncontrollability to weighted digraphs with multipleleaders and including broadcasted control. Our results show that the internal structure of communities in a graph can induce obstructions to controllability that cannot be characterized by symmetry arguments alone and that in some cases depend on the number-theoretic properties of the communities. We show via examples that our results can be used to account for a large portion of uncontrollable inducing leader-selections that could not have otherwise been accounted for using symmetry results.
\end{abstract}

Key words: Networked Control Systems; Network Controllability; Equitable Partitions

\section{Introduction}

The controllability property of a controlled dynamical system is one of the central notions in control systems theory. Roughly speaking, the controllability problem is concerned with whether it is possible to transfer the state of a controlled dynamic system from some given initial condition to any final desired state. For linear control systems, there are several equivalent characterizations of controllability and it is well-known that the property is generic in the sense that the set of systems that are controllable form an open and dense subset of the space of all system parameters. Despite the generic nature of controllability for linear systems, there has been recent interest in the control community to understand the controllability property for linear multi-agent networked control systems, see for instance $[26,24,16,23,28,22,5,1,19]$ and references therein. The goal of this recent research effort is to understand how graph-theoretic properties of the underlying network relate to the controllability property of the linear control system whose system matrix encodes the adjacency relationships in the network (e.g., adjacency matrix, Laplacian matrix, etc.).

\footnotetext{
* This paper was not presented at any IFAC meeting. The first author acknowledges the support of the National Science Foundation under Grant No. ECCS-1700578.

Email addresses: aguilar@geneseo.edu (Cesar O. Aguilar), bahman@mast.queensu.ca (Bahman Gharesifard).
}

It is natural to ask why such an effort has been devoted to characterize a generic property such as controllability for a linear control system. To answer this question, we first note that the generic nature of controllability when system parameters are allowed to vary continuously does not in any trivial way imply that controllability is also generic for discrete combinatorial objects such as graphs. The situation is similar, for instance, when considering the question of the likelihood that a matrix will have simple eigenvalues. If the matrix entries are allowed to vary continuously then the set of real matrices with simple eigenvalues forms a dense and open subset of the associated Euclidean space. In contrast, up until very recently and using sophisticated machinery, it was shown that the adjacency matrix of a graph will almost surely have simple eigenvalues as the size of the graph increases [27], and thus settling a conjecture posed by L. Babai in the 1980s. Similarly, it was also shown very recently that the controllability property for the adjacency matrix of a graph, and in the special case where all nodes are controlled, is generic, again in the sense that the proportion of controllable systems tends to one as the size of the graph increases (thus settling a conjecture posed by C. Godsil [10]). Second of all, it has been documented in [15] that many real-world biological, technological, and social networks possess a high level of symmetry and, as it was shown in [24], symmetries are obstructions to controllability for networked multi-agent control systems. As pointed out in [15], many real-world 
networks contain "tree-like" symmetries due to the fact that these networks are grown from existing vertices and this growth process introduces branch-like structures. Since almost all tree networks have symmetries [8], it is not too surprising then that such symmetries would be present in these networks. Hence, although symmetrylike structures are mathematically rare, they seem to be ubiquitous in many real-world networks and therefore it is important to uncover what other symmetry-like structures induce uncontrollability.

The purpose of this paper is to identify a symmetry-like structure, specifically a class of graph vertex partitions, that when present in a network can obstruct controllability in a multi-agent control system even when the control nodes have been selected to "break" all symmetries. It is well-known that symmetries are not necessary for uncontrollability [24] but aside from special classes of graphs such as trees, grid graphs, threshold graphs, and circulant graphs $[13,22,21,2]$, and a linear-algebraic characterization of controllability for multi-agent systems [4], little is known about what general intrinsic graph-structures induce uncontrollability. A purpose of this paper is to narrow this gap.

As mentioned, we identify a class of graph vertex partitions, and in particular a class of almost equitable partitions [3], that induce uncontrollability in a non-trivial way. Moreover, these partitions can account for a significant portion of the uncontrollable leader selections that could not have been detected by symmetry arguments alone. Using graph vertex partitions to study controltheoretic properties in multi-agent systems is not new and in fact is becoming an increasingly useful tool in the analysis and design of multi-agent control systems, see for instance $[24,16,28,18,20]$ and references therein. Graph vertex partitions also take an important role in the study of synchrony and pattern formation in coupled cell networks $[25,12]$.

\section{Statement of Contributions}

The main results of this paper are new general graphtheoretic necessary conditions for controllability of leader-follower Laplacian dynamics using almost equitable graph partitions (Theorems 3, 4, 5). Our first main result (Theorem 3) generalizes the relationship between graph symmetries and uncontrollability in the multi-input case [24] to also include the scenario of multi-input broadcast control. Roughly speaking, this result shows that if the nodes are selected to respect the structure of a non-trivial almost equitable partition then the resulting control system is uncontrollable. Our next main result (Theorem 4) identifies a new obstruction to controllability that we call reducible equitable partitions. These partitions are, to the best knowledge of the authors, the first general and intrinsic graph-theoretic structure that induce uncontrollability unrelated to graph symmetries. In other words, when these partitions are present, one might have chosen the leaders to break all "symmetries" induced by all non-trivial almost equitable partitions but yet the induced dynamics are uncontrollable. The result demonstrates that the internal structure of the cells of a partition can also introduce undesirable controllability properties. Our last main result (Theorem 5) uses the quotient graph induced by an almost equitable partition to identify a new obstruction to controllability that again cannot be captured by symmetry or using reducible equitable partitions. This result also demonstrates that the internal structure of the cells of a partition must be taken into account in controllability analysis. Overall, our results provide explicit graph-theoretic structures that when present in a network can induce uncontrollable dynamics.

Although we consider Laplacian dynamics, our results are applicable to other graph matrices that encode the adjacency structure of a graph. Also, we focus on the broadcasted control problem since it allows us to broadened the view of how symmetries, and more generally equitable partitions, induce uncontrollability and allows us to identify new obstructions to controllability that could not have been captured otherwise. We note that the broadcasted multi-agent control scenario is also considered in $[10,14]$.

\section{Preliminaries and Problem Statement}

Throughout this paper, the standard basis vectors in $\mathbb{R}^{n}$ are denoted by $\mathbf{e}_{1}, \mathbf{e}_{2}, \ldots, \mathbf{e}_{n}$. The orthogonal complement of a set $S \subset \mathbb{R}^{n}$ under the standard inner product on $\mathbb{R}^{n}$ will be denoted by $S^{\perp}$. The transpose of a matrix $\mathbf{M}$ is denoted by $\mathbf{M}^{T}$. The $n \times n$ identity matrix is denoted by $\mathbf{I}_{n}$. The conjugate transpose of $\mathbf{w} \in \mathbb{C}^{n}$ is denoted by $\mathbf{w}^{*}$. The column space and null space of a matrix $\mathbf{M}$ will be denoted by $\operatorname{img}(\mathbf{M})$ and $\operatorname{ker}(\mathbf{M})$, respectively. The cardinality of a finite set $C$ will be denoted by $|C|$. The greatest common divisor of a set of integers $k_{1}, k_{2}, \ldots, k_{r}$ is denoted by $\operatorname{gcd}\left(k_{1}, k_{2}, \ldots, k_{r}\right)$.

\subsection{Permutations}

We denote the symmetric group on $V=\{1,2, \ldots, n\}$ by $S_{n}$, i.e., $S_{n}$ is the group of permutations $\sigma: V \rightarrow V$. It is well-known [7] that each $\sigma \in S_{n}$ has a unique (up to ordering) cycle decomposition of the form

$$
\begin{aligned}
\sigma=\underbrace{\left(i_{1} i_{2} \cdots i_{m_{1}}\right.}_{\rho_{1}}) & \underbrace{\left(i_{m_{1}+1} i_{m_{1}+2} \cdots i_{m_{2}}\right)}_{\rho_{2}} \\
& \cdots \underbrace{\left(i_{m_{r-1}+1} i_{m_{r-1}+2} \cdots i_{m_{r}}\right.}_{\rho_{r}})
\end{aligned}
$$

where $\rho_{j}$ is the permutation that cyclically permutes the integers $i_{m_{j-1}+1}, i_{m_{j-1}+2}, \ldots, i_{m_{j}}$ and fixes all other integers. The set of integers $C_{j}=$ $\left\{i_{m_{j-1}+1}, i_{m_{j-1}+2}, \ldots, i_{m_{j}}\right\}$ is called a cell.

The set of binary vectors of length $n$ will be denoted by $\{0,1\}^{n}$. The characteristic indices of $\mathbf{b} \in\{0,1\}^{n}$, denoted by $\chi(\mathbf{b})$, is the subset of indices where $\mathbf{b}$ is nonzero. For instance, if $\mathbf{b}=\left[\begin{array}{llllll}0 & 1 & 1 & 0 & 0 & 1\end{array}\right]^{T} \in\{0,1\}^{6}$ then $\chi(\mathbf{b})=\{2,3,6\}$. Conversely, given any set $C \subseteq$ $\{1,2, \ldots, n\}$ of indices, the characteristic vector of $C$ is the unique vector $\mathbf{c} \in\{0,1\}^{n}$ such that $\chi(\mathbf{c})=C$. The 
all ones vector and the zero vector will be denoted by $\mathbf{1}_{n}$ and $\mathbf{0}_{n}$, respectively.

\subsection{Graphs}

Our notation from graph theory is standard and any basic notion not defined here can be found in [11]. In this paper, we consider graphs that may be weighted and/or directed, but not containing loops. For a (directed) graph $G$, or digraph, we denote by $V(G)$ its vertex set and by $E(G) \subseteq V(G) \times V(G)$ its edge set. We consider only finite graphs and so we assume throughout that $V(G)=$ $\{1,2, \ldots, n\}$. The weight of the edge $(i, j) \in E(G)$ will be denoted by $a_{i j} \in \mathbb{R}$, and if $(i, j) \notin E(G)$ we set $a_{i j}=0$. When not explicitly stated, we assume that $G$ is strongly connected.

The out-neighbors of $i \in V$ is the set of vertices $\mathcal{N}_{\text {out }}(i):=\{j \in V \mid(i, j) \in E\}$ and the out-degree of $i$ is

$$
d_{\text {out }}(i)=\sum_{j \in \mathcal{N}_{\text {out }}(i)} a_{i j}=\sum_{j \in V} a_{i j} .
$$

The out-adjacency matrix of $G$ is the $n \times n$ matrix $\mathbf{A}$ defined as $(\mathbf{A})_{i j}=a_{i j}$. The out-degree matrix of $G$ is the $n \times n$ diagonal matrix $\mathbf{D}$ defined by $(\mathbf{D})_{i i}=$ $d_{\text {out }}(i)$. The out-Laplacian matrix of $G$ is the matrix $\mathbf{L}=\mathbf{D}-\mathbf{A}$. Since $G$ is strongly connected, $\lambda=0$ is a simple eigenvalue of $\mathbf{L}$ affording the eigenvector $\mathbf{1}_{n}$. Henceforth, by adjacency and Laplacian matrix we mean the out-adjacency and out-Laplacian matrix. The in-neighbors, in-degree, in-adjacency, and in-Laplacian matrix are defined similarly. When $G$ is not directed, $d_{i}:=d_{\text {out }}(i)=d_{\text {in }}(i)$, for all $i \in V$.

A permutation $\sigma \in S_{n}$ is an automorphism of $G$ if $(i, j) \in$ $E(G)$ if and only if $(\sigma(i), \sigma(j)) \in E(G)$. The group of automorphisms of $G$ will be denoted by $\operatorname{Aut}(G) \subset S_{n}$. We say that $G$ is asymmetric if $\operatorname{Aut}(G)$ contains only the identity permutation, and is called symmetric otherwise.

\subsection{Linear Algebra}

We briefly recall some basic facts from linear algebra that will be used frequently. Let $\mathrm{V}$ be an $n$-dimensional inner product space, let $\mathrm{T}: \mathrm{V} \rightarrow \mathrm{V}$ be a diagonalizable linear operator, and let $\mathrm{W} \subset \mathrm{V}$ be a $k$-dimensional subspace. It is well-known that $\mathrm{V}=\mathrm{W} \oplus \mathrm{W}^{\perp}$, that is, $\mathrm{W} \cap \mathrm{W}^{\perp}=\{0\}$ and $\mathrm{V}=\mathbf{W}+\mathbf{W}^{\perp}:=\left\{w+w^{\prime} \mid w \in \mathbf{W}, w^{\prime} \in \mathbf{W}^{\perp}\right\}$. We say that $\mathbf{W}$ is $\mathbf{T}$-invariant if $\mathbf{T}(w) \in \mathbf{W}$ for every $w \in \mathbf{W}$. In this case, one can show that $W^{\perp}$ is $T^{*}$-invariant, where $\mathrm{T}^{*}$ denotes the adjoint of $\mathrm{T}$. In addition, if $\mathrm{T}$ is selfadjoint, there exists a basis of $\mathrm{V}$ consisting of eigenvectors $\left\{\mathbf{v}_{1}, \mathbf{v}_{1}, \ldots, \mathbf{v}_{n}\right\}$ of $\mathrm{T}$, where $\mathbf{v}_{1}, \ldots, \mathbf{v}_{k} \in \mathrm{W}$ and $\mathbf{v}_{k+1}, \ldots, \mathbf{v}_{n} \in \mathrm{W}^{\perp}$. In other words, the decomposition $\mathrm{V}=\mathrm{W} \oplus \mathrm{W}^{\perp}$ induces a splitting of the eigenvectors of $\mathrm{T}$ so that some eigenvectors will be contained in $\mathrm{W}$ and the rest will be contained in $\mathrm{W}^{\perp}$. The properties of $\mathrm{T}$-invariance and self-adjointness are critical here, since even when $T$ is diagonalizable this is not generally true for an arbitrary subspace $\mathrm{W}$.

\subsection{The Graph Controllability Problem}

Let $\mathbf{L}$ be the Laplacian matrix of a weighted digraph $G$ on $n$ vertices. The leader-selection controllability problem for $\mathbf{L}$ with $m \geq 1$ inputs is to find a matrix $\mathbf{B} \in$ $\{0,1\}^{n \times m}$ such that the linear control system

$$
\dot{\mathbf{x}}=-\mathbf{L x}+\mathbf{B u}
$$

is controllable. It is well-known that the pair $(\mathbf{L}, \mathbf{B})$ is controllable if and only if the smallest $\mathbf{L}$-invariant subspace containing $\operatorname{img}(\mathbf{B})$, which we denote throughout by $\langle\mathbf{L} ; \mathbf{B}\rangle$, is all of $\mathbb{R}^{n}$. An equivalent characterization of controllability is the Popov-Belevitch-Hautus (PBH) eigenvector test [6].

Theorem 1 (PBH) The linear control system $(\mathbf{L}, \mathbf{B})$ is uncontrollable if and only if there exists an eigenvector $\mathbf{w} \in \mathbb{C}^{n}$ of $\mathbf{L}^{T}$ such that $\mathbf{w}^{*} \mathbf{B}=\mathbf{0}_{1 \times m}$.

In this paper, we are mostly interested in the broadcast single-input controllability problem. In this scenario, there is a single control input broadcasted to a selected number of nodes in the network. These selected nodes are called the leaders and the remaining nodes are called the followers. The single-input controllability problem amounts to the case that $\mathbf{B}$ is a binary vector and thus in this case, we write $\mathbf{b}$ instead of $\mathbf{B}$. However, one of our main results (Theorem 3 ) is stated for the multi-input case and the our results for the single-input case (Theorem 4 and Theorem 5) can be extended to the multi-input case. We leave this for a future paper.

\section{Almost Equitable Partitions of Weighted Di- graphs}

In this section, we introduce the main intrinsic graphtheoretic structure that we use, namely almost equitable partitions, and related theorems. Let $G$ be a weighted digraph with adjacency matrix $\mathbf{A}=\left(a_{i j}\right)$. Given a subset $C \subseteq V$, we denote for each $i \in V$ the out-degree of $i$ relative to $C$ by $d_{\text {out }}(i, C)=\sum_{j \in C} a_{i j}$. Let $\pi=$ $\left\{C_{1}, C_{2}, \ldots, C_{k}\right\}$ be a partition of the vertex set $V$, that is, $\bigcup_{i=1}^{k} C_{i}=V$ and $C_{i} \cap C_{j}=\emptyset$ for $i \neq j$. The characteristic matrix of $\pi$ is the $n \times k$ matrix $\mathbf{P}(\pi)$ whose $j$ th column is the characteristic vector of the cell $C_{j}$. Clearly, $\operatorname{img}(\mathbf{P}(\pi))$ is a $k$-dimensional subspace of $\mathbb{R}^{n}$ consisting of vectors that are constant on the cells $C_{1}, \ldots, C_{k}$, that is, if $\mathbf{x} \in \operatorname{img}(\mathbf{P}(\pi))$ then the components of $\mathbf{x}$ on $C_{j}$ are all equal for all $C_{j}$. When $\pi$ is understood, we will write $\mathbf{P}$ instead of $\mathbf{P}(\pi)$. Following [3], we give the next definition.

Definition 1 Let $G$ be a graph with vertex set $V$ and let $\pi=\left\{C_{1}, C_{2}, \ldots, C_{k}\right\}$ be a partition of $V$. We call $\pi$ an almost equitable partition (AEP) of $G$ if for all distinct ordered pairs of cells $\left(C_{r}, C_{s}\right)$ it holds that $d_{\text {out }}\left(i, C_{s}\right)=$ $d_{\text {out }}\left(j, C_{s}\right)$, for all $i, j \in C_{r}$.

In other words, $\pi$ is an AEP of $G$ if every vertex in $C_{r}$ has the same number of neighbors in $C_{s}$, and this holds for every ordered pair $\left(C_{r}, C_{s}\right)$. In this case, we denote by $\alpha_{r s}:=d_{\text {out }}\left(i, C_{s}\right)$, for any $i \in C_{r}$. We note that, in general, $\alpha_{r s} \neq \alpha_{s r}$. We define $\alpha_{r r}=0$, for all $r=1,2, \ldots, k$. 
We note that the partitions $\pi=\{\{1,2,3, \ldots, n\}\}$ and $\pi=\{\{1\},\{2\}, \ldots,\{n\}\}$ are trivially almost equitable partitions.

Remark 1 An almost equitable partition is called an equitable partition if $d_{\text {out }}\left(i, C_{r}\right)=d_{\text {out }}\left(j, C_{r}\right)$ for every $i, j \in C_{r}$. In other words, every vertex in the same cell has the same number of neighbors within its own cell. •

As the next lemma states, the cells in the cycle decomposition (see Section 2.1) of an automorphism of a graph induces an equitable partition, and therefore any result on almost equitable partitions is directly applicable to the partitions induced by an automorphism. The straightforward proof of the following is left to the reader.

Lemma 1 Let $G$ be a graph and suppose that the cycle decomposition of $\sigma \in \operatorname{Aut}(G)$ induces the partition $\pi=$ $\left\{C_{1}, C_{2}, \ldots, C_{k}\right\}$ of $V$. Then $\pi$ is an equitable partition of $G$.

Given an almost equitable partition $\pi$ of $G$, we define the out-neighbor quotient graph of $G$ over $\pi$, denoted by $G / \pi$, as the weighted digraph with vertices $V(G / \pi)=\pi=$ $\left\{C_{1}, C_{2}, \ldots, C_{k}\right\}$, edge set $E(G / \pi)=\left\{\left(C_{r}, C_{s}\right) \mid \alpha_{r s} \neq\right.$ $0\}$, and the weight of $\left(C_{r}, C_{s}\right) \in E(G / \pi)$ is $\alpha_{r s}$. We let $\mathbf{A}_{\pi}$ and $\mathbf{L}_{\pi}$ denote the out-adjacency and out-Laplacian matrix of $G / \pi$, respectively. In particular, for $r \neq s$, we have that $\left(\mathbf{L}_{\pi}\right)_{r, s}=-\alpha_{r, s}$, and $\left(\mathbf{L}_{\pi}\right)_{r, r}=d_{\text {out }}\left(C_{r}\right)=$ $\sum_{C_{s} \in V(G / \pi)} \alpha_{r, s}$. In general, $G / \pi$ is a weighted digraph even when $G$ is unweighted and undirected.

The following theorem describes one way in which $\mathbf{L}$ invariant subspaces arise with respect to an almost equitable partition. The proof of the following is similar to [3] and thus omitted.

Theorem 2 Let $G$ be a weighted digraph with outLaplacian matrix $\mathbf{L}$, let $\pi=\left\{C_{1}, C_{2}, \ldots, C_{k}\right\}$ be a partition of $V(G)$, and let $\mathbf{P}=\mathbf{P}(\pi)$. Then $\pi$ is an almost equitable partition of $G$ if and only if $\operatorname{img}(\mathbf{P})$ is $\mathbf{L}$-invariant. In this case, $\mathbf{L}_{\pi}=\left(\mathbf{P}^{T} \mathbf{P}\right)^{-1} \mathbf{P}^{T} \mathbf{L P}$ and $\mathbf{L P}=\mathbf{P L}_{\pi}$.

Hence, when $\pi$ is an almost equitable partition, $\mathbf{L}_{\pi}$ is the matrix representation of the restriction of $\mathbf{L}$ to the subspace $\mathbf{W}=\operatorname{img}(\mathbf{P}(\pi))$ in the basis obtained from the columns of $\mathbf{P}(\pi)$. We note that even if $\mathbf{L}$ is symmetric, $\mathbf{L}_{\pi}$ is not generally symmetric since the columns of $\mathbf{P}(\pi)$ are not an orthonormal basis of W. However, $\left.\mathbf{L}\right|_{\mathbf{W}}$ is indeed self-adjoint if $\mathbf{L}$ is self-adjoint. In any case, every eigenvector of $\left.\mathbf{L}\right|_{W}$ can be identified with an eigenvector of $\mathbf{L}$ as the next results states.

Lemma 2 Let $G, \mathbf{L}, \mathbf{L}_{\pi}$, and $\mathbf{P}$ be as in Theorem 2. Then $(\mathbf{v}, \lambda)$ is an eigenvector-eigenvalue pair of $\mathbf{L}_{\pi}$ if and only if $(\mathbf{P v}, \lambda)$ is an eigenvector-eigenvalue pair of $\mathbf{L}$.

To end this section, we prove the next result that describes how AEPs of the quotient graph induce AEPs of the original graph.

Proposition 1 Let $G$ be a weighted digraph and suppose that $\pi=\left\{C_{1}, C_{2}, \ldots, C_{k}\right\}$ is an AEP of $G$. Suppose that $\rho=\left\{S_{1}, S_{2}, \ldots, S_{\ell}\right\}$ is an $A E P$ of the quotient graph $G / \pi$. Define the partition $\pi / \rho=\left\{\bar{C}_{1}, \bar{C}_{2}, \ldots, \bar{C}_{\ell}\right\}$ of
$V(G)$ by $\bar{C}_{j}:=\bigcup_{C_{i} \in S_{j}} C_{i}$ for $j=1,2, \ldots, \ell$. Then $\pi / \rho$ is an almost equitable partition of $G$.

PROOF. It is easy to see that the characteristic matrix of $\pi / \rho$ is $\mathbf{P}(\pi / \rho)=\mathbf{P}(\pi) \mathbf{P}(\rho)$. Let now $\mathbf{L}_{\pi / \rho}$ be the Laplacian matrix of the quotient graph $(G / \pi) / \rho$. Then, by Theorem $2, \mathbf{L}_{\pi} \mathbf{P}(\rho)=\mathbf{P}(\rho) \mathbf{L}_{\pi / \rho}$, since $\rho$ is an AEP of $G / \pi$. Then

$$
\begin{aligned}
\mathbf{L P}(\pi / \rho)=\mathbf{L P}(\pi) \mathbf{P}(\rho) & =\mathbf{P}(\pi) \mathbf{L}_{\pi} \mathbf{P}(\rho) \\
& =\mathbf{P}(\pi) \mathbf{P}(\rho) \mathbf{L}_{\pi / \rho} \\
& =\mathbf{P}(\pi / \rho) \mathbf{L}_{\pi / \rho}
\end{aligned}
$$

Hence, $\operatorname{img}(\mathbf{P}(\pi / \rho))$ is $\mathbf{L}$-invariant and Theorem 2 implies that $\pi / \rho$ is an AEP of $G$.

In Proposition 1, the partition $\pi / \rho$ is a coarsening of the partition $\pi$, i.e., every cell in $\pi$ is a subset of a cell in $\pi / \rho$. We illustrate Proposition 1 with an example that will be used throughout the paper.

Example 1 Let $G$ be the unweighted and undirected graph shown in Figure 1. The automorphism group $\operatorname{Aut}(G)$, as computed by NAUTY [17], is generated by the permutations $\tau_{1}=\left(\begin{array}{ll}2 & 3\end{array}\right), \tau_{2}=\left(\begin{array}{ll}7 & 8\end{array}\right)$, $\tau_{3}=\left(\begin{array}{l}1 \\ 6\end{array}\right)$, and $\tau_{4}=\left(\begin{array}{l}4 \\ 5\end{array}\right)$. By Lemma 1, the automorphism $\sigma=\tau_{1} \tau_{2} \tau_{3} \tau_{4}$ induces an equitable partition $\pi=\left\{C_{1}, C_{2}, C_{3}, C_{4}, C_{5}, C_{6}\right\}$ of $V(G)$ whose cells are $C_{1}=\{2,3\}, C_{2}=\{7,8\}, C_{3}=\{1,6\}, C_{4}=\{4,5\}$, $C_{5}=\{9\}$, and $C_{6}=\{10\}$. The quotient graph $G / \pi$ is shown in Figure 2, where the weights shown on the edges correspond to the out-degree of the nodes. It can be verified that $\rho=\{\{3,4\},\{5,6\},\{1\},\{2\}\}$ is an equitable partition of $G / \pi$ induced by the automorphism $\rho=\left(\begin{array}{ll}3 & 4\end{array}\right)\left(\begin{array}{ll}5 & 6\end{array}\right)$ of $G / \pi$. The quotient graph $(G / \pi) / \rho$ is displayed in Figure 3. The partition $\pi / \rho$ is therefore

$$
\pi / \rho=\{\{2,3\},\{7,8\},\{9,10\},\{1,6,4,5\}\},
$$

which as expected is coarser than $\pi$. It can be verified that $\pi / \rho$ is indeed an almost equitable partition of $G$ but not an equitable partition. Hence, although $\pi$ and $\rho$ are equitable partitions, $\pi / \rho$ may in general be only almost equitable.

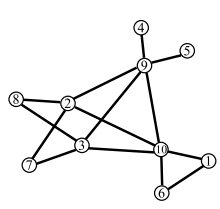

Fig. 1. $G$

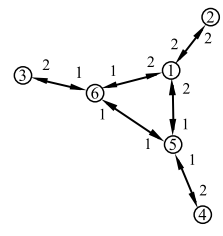

Fig. 2. $G / \pi$

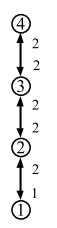

Fig. 3. $(G / \pi) / \rho$

\section{Controllability and Almost Equitable Parti-} tions

In this section, we present our main results relating controllability with almost equitable partitions. 
Suppose that $\pi$ is an almost equitable partition of $G$ and let $\mathbf{P}=\mathbf{P}(\pi)$ be the characteristic matrix of $\pi$. Then, since $\mathbf{W}=\operatorname{img}(\mathbf{P})$ is $\mathbf{L}$-invariant, it follows that $\mathbf{W}^{\perp}=\operatorname{ker}\left(\mathbf{P}^{T}\right)$ is $\mathbf{L}^{T}$-invariant. If $G$ is undirected (this assumption is not needed but simplifies the forthcoming discussion) then $\mathbf{L}$ is a symmetric matrix and hence $\operatorname{ker}\left(\mathbf{P}^{T}\right)$ is also $\mathbf{L}$-invariant (see Section 2.3). From the direct-sum decomposition $\mathbb{R}^{n}=\operatorname{img}(\mathbf{P}) \oplus \operatorname{ker}\left(\mathbf{P}^{T}\right)$, we may therefore partition the eigenvectors of $\mathbf{L}$ into those that are contained in $\operatorname{img}(\mathbf{P})$ and those that are contained in $\operatorname{ker}\left(\mathbf{P}^{T}\right)$ (see Section 2.3). Now, vectors in $\operatorname{img}(\mathbf{P})$ are constant on the cells of $\pi$ and vectors in $\operatorname{ker}\left(\mathbf{P}^{T}\right)$ sum to zero on the cells of $\pi$. Hence, if the leader nodes are selected so that the resulting control input matrix has columns that are constant on the cells of $\pi$, these columns will be orthogonal to every eigenvector of $\mathbf{L}$ in $\operatorname{ker}\left(\mathbf{P}^{T}\right)$ and thus by the $\mathrm{PBH}$ test the system is uncontrollable. The following result builds on our observations in the preceding discussion and considers the general directed case.

Theorem 3 Let $G$ be a weighted digraph on $n \geq 2$ vertices and suppose that $\pi=\left\{C_{1}, C_{2}, \ldots, C_{k}\right\}$ is an almost equitable partition of $G$ with characteristic matrix $\mathbf{P}$, where $1 \leq k<n$. Let $\mathbf{b}_{1}, \ldots, \mathbf{b}_{m} \in\{0,1\}^{n}$ and assume that each $\mathbf{b}_{i}$ is constant on the cells of $\pi$, and put $\mathbf{B}=\left[\begin{array}{llll}\mathbf{b}_{1} & \mathbf{b}_{2} & \cdots & \mathbf{b}_{m}\end{array}\right]$. Then (i) $\langle\mathbf{L} ; \mathbf{B}\rangle \subseteq \operatorname{img}(\mathbf{P})$, (ii) $\operatorname{dim}\langle\mathbf{L}, \mathbf{B}\rangle \leq k$, (iii) there exists an eigenvector $\mathbf{w}$ of $\mathbf{L}^{T}$ contained in $\operatorname{ker}\left(\mathbf{P}^{T}\right)$ such that $\mathbf{w}^{*} \mathbf{B}=\mathbf{0}_{1 \times m}$, and (iv) $(\mathbf{L}, \mathbf{B})$ is uncontrollable.

PROOF. Since $\mathbf{b}_{1}, \ldots, \mathbf{b}_{m}$ are constant on the cells of $\pi$, it follows that $\operatorname{img}(\mathbf{B}) \subseteq \operatorname{img}(\mathbf{P})$. By Theorem 2, $\operatorname{img}(\mathbf{P})$ is $\mathbf{L}$-invariant and thus since $\langle\mathbf{L} ; \mathbf{B}\rangle$ is the smallest $\mathbf{L}$-invariant subspace containing $\operatorname{img}(\mathbf{B})$, we must have $\langle\mathbf{L} ; \mathbf{B}\rangle \subseteq \operatorname{img}(\mathbf{P})$, and therefore $\operatorname{dim}\langle\mathbf{L}, \mathbf{B}\rangle \leq k$. Since $k<n$ then $(\mathbf{L}, \mathbf{B})$ is uncontrollable. Since $\operatorname{ker}\left(\mathbf{P}^{T}\right)$ is a non-trivial invariant subspace of $\mathbf{L}^{T}$ there is at least one eigenvector $\mathbf{w} \in \mathbb{C}^{n}$ of $\mathbf{L}^{T}$ contained in $\operatorname{ker}\left(\mathbf{P}^{T}\right)=$ $\operatorname{img}(\mathbf{P})^{\perp}$, and thus $\mathbf{w}^{*} \mathbf{B}=\mathbf{0}_{1 \times m}$.

Remark 2 The result in [28, Thm. 2] is a special case of Theorem 3 , since there $\mathbf{B}=\left[\begin{array}{lll}\mathbf{e}_{i_{1}} & \cdots & \mathbf{e}_{i_{m}}\end{array}\right]$ and the first $m$ cells of $\pi$ are $C_{j}=\left\{i_{j}\right\}, 1 \leq j \leq m$, so that Theorem 3 is applicable.

Theorem 3 contains as a special case the situation of leader-symmetric selections introduced in [24].

Definition 2 Let $G$ be a weighted digraph. We say that $\mathbf{B} \in\{0,1\}^{n \times m}$ is leader-symmetric if there exists a nontrivial automorphism $\sigma \in \operatorname{Aut}(G)$ such that $\operatorname{img}(\mathbf{B}) \subseteq$ $\operatorname{img}(\mathbf{P})$, where $\mathbf{P}$ is the characteristic matrix of the partition of $V(G)$ induced by the automorphism $\sigma$.

Corollary 1 Let $G$ be a weighted digraph on $n \geq 2$ vertices. If $\mathbf{B} \in\{0,1\}^{n \times m}$ is leader-symmetric then $(\mathbf{L}, \mathbf{B})$ is an uncontrollable pair.

Remark 3 Proposition IV.8 in [5] is a special case of Corollary 1 since in [5] we have $\mathbf{B}=\left[\begin{array}{lll}\mathbf{e}_{i_{1}} & \cdots & \mathbf{e}_{i_{m}}\end{array}\right]$ and leader-symmetry amounts to the existence of a non-

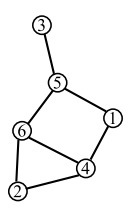

Fig. 4. An asymmetric graph with non-trivial almost equitable partitions.

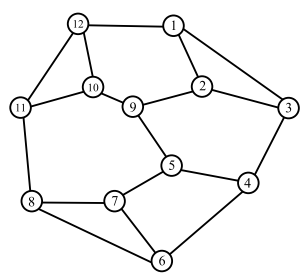

Fig. 5. Frucht graph trivial automorphism $\sigma$ such that $\sigma\left(i_{j}\right)=i_{j}$ for all $j=$ $1, \ldots, m$. In this case, the equitable partition induced by $\sigma$ contains the singleton cells $\left\{i_{1}\right\},\left\{i_{2}\right\}, \ldots,\left\{i_{m}\right\}$, so that $\mathbf{B}$ is trivially constant on the cells of $\sigma$. This shows that by not considering the broadcast control problem one cannot detect intrinsic graph structures that are characterized by vertex partitions whose cells all contain more than one vertex. Also, from a practical view-point, applying the same control at $m$ nodes is preferable over applying distinct independent controls at $m$ nodes.

Theorem 3 can be seen as a natural generalization of the statement that "symmetry" in a graph is a potential obstruction to controllability. Of course, a graph may have a trivial automorphism group but still have non-trivial almost equitable partitions. The following example illustrates this scenario where Theorem 3 is applicable.

Example 2 Consider the graph $G$ on $n=6$ vertices shown in Figure 4. This graph is asymmetric but there are 14 non-trivial binary vectors $\mathbf{b} \in\{0,1\}^{6}$ that induce an uncontrollable pair $(\mathbf{L}, \mathbf{b})\left(\mathbf{b}=\mathbf{0}_{n}\right.$ and $\mathbf{b}=\mathbf{1}_{n}$ trivially induce uncontrollability). Hence, Corollary 1 cannot be used to characterize these uncontrollable scenarios. However, two of the 14 can be explained by Theorem 3. To see this, it is straightforward to verify that $\pi=\{\{5,6\},\{1,2,3,4\}\}$ is an almost equitable partition of $G$. Therefore, by Theorem 3 , the leader selections $\mathbf{b}_{1}=\left[\begin{array}{llllll}0 & 0 & 0 & 0 & 1 & 1\end{array}\right]^{T}$ and $\mathbf{b}_{2}=\left[\begin{array}{llllll}1 & 1 & 1 & 1 & 0 & 0\end{array}\right]^{T}$ induce uncontrollable dynamics, since clearly they are contained in $\operatorname{img}(\mathbf{P}(\pi))$, where in this case $\mathbf{P}(\pi)=\left[\begin{array}{ll}\mathbf{b}_{1} & \mathbf{b}_{2}\end{array}\right]$.

A natural question to ask then is whether uncontrollability of $(\mathbf{L}, \mathbf{B})$ is due to $\operatorname{img}(\mathbf{B}) \subset \operatorname{img}(\mathbf{P})$, where $\mathbf{P}$ is the characteristic matrix of some non-trivial almost equitable partition. In other words, what are other intrinsic graph-theoretic structures that induce uncontrollable leader selections? To shed light into this question, and to simplify the forthcoming presentation, we consider single-input leader selections $\mathbf{b} \in\{0,1\}^{n}$ and undirected graphs.

Example 3 Consider again the graph $G$ on $n=6$ vertices shown in Figure 4, which has the AEP $\pi=\{\{5,6\},\{1,2,3,4\}\}$, and let $C_{1}=\{5,6\}$ and $C_{2}=\{1,2,3,4\}$ be the cells of $\pi$. By Theorem 3, the leaders selection $\mathbf{e}_{5}+\mathbf{e}_{6}$, and its binary complement $\mathbf{e}_{1}+\mathbf{e}_{2}+\mathbf{e}_{3}+\mathbf{e}_{4}$, yield uncontrollable dynamics. Let $\mathbf{w} \neq \mathbf{1}_{n}$ be an eigenvector of $\mathbf{L}$ contained in $\operatorname{img}(\mathbf{P}(\pi))$. Hence, $\mathbf{w}=\alpha_{1} \mathbf{c}_{1}+\alpha_{2} \mathbf{c}_{2}$, where $\mathbf{c}_{1}=\mathbf{e}_{5}+\mathbf{e}_{6}$ and $\mathbf{c}_{2}=\mathbf{e}_{1}+\mathbf{e}_{2}+\mathbf{e}_{3}+\mathbf{e}_{4}$ are the columns of $\mathbf{P}(\pi)$. 
Since $\mathbf{w}$ and $\mathbf{1}_{n}$ are eigenvectors of the symmetric matrix $\mathbf{L}$, it follows that $\mathbf{w}^{T} \mathbf{1}_{n}=0$ and therefore $\left|C_{1}\right| \alpha_{1}+\left|C_{2}\right| \alpha_{2}=2 \alpha_{1}+4 \alpha_{2}=0$, or equivalently $\alpha_{1}+2 \alpha_{2}=0$. The reduced relation $\alpha_{1}+2 \alpha_{2}=0$ induces new uncontrollable leader selections not contained in $\operatorname{img}(\mathbf{P}(\pi))$, that is, not characterized by Theorem 3. To see this, suppose that $\mathbf{b}$ is chosen so that $\left|\chi(\mathbf{b}) \cap C_{1}\right|=1$ and $\left|\chi(\mathbf{b}) \cap C_{2}\right|=2$. For example, if $\mathbf{b}=\left[\begin{array}{llllll}1 & 0 & 1 & 0 & 0 & 1\end{array}\right]^{T}$ then $\chi(\mathbf{b})=\{1,3,6\}$. By construction, $\mathbf{b}$ is not in $\operatorname{img}(\mathbf{P}(\pi))$, and clearly $\mathbf{w}^{T} \mathbf{b}=\left|\chi(\mathbf{b}) \cap C_{1}\right| \alpha_{1}+\left|\chi(\mathbf{b}) \cap C_{2}\right| \alpha_{2}=0$. Then by the $\mathrm{PBH}$ test, $(\mathbf{L}, \mathbf{b})$ is uncontrollable. There are $\left(\begin{array}{l}2 \\ 1\end{array}\right)\left(\begin{array}{l}4 \\ 2\end{array}\right)=12$ such choices for $\mathbf{b}$, and with the 2 uncontrollable leader selections in $\operatorname{img}(\mathbf{P}(\pi))$ characterized by Theorem 3, this yields a total of 14 non-trivial uncontrollable leader selections. This completely characterizes all the uncontrollable leader selections for the graph in Figure 4.

The previous example illustrates how the internal local structure of the cells, or communities, of a partition of the vertex set $V(G)$ can introduce new obstructions to controllability that cannot be characterized by symmetry arguments. The example shows that although all symmetries of a graph can be broken by an appropriate choice of leader nodes, one must still take care in not preserving any internal local structure of the communities in a network. To formalize the previous example, we introduce the following definition.

Definition 3 Let $G$ be a graph and suppose that $\pi=$ $\left\{C_{1}, C_{2}, \ldots, C_{k}\right\}$ is a partition of $V(G)$. We define

$$
\operatorname{gcd}(\pi):=\operatorname{gcd}\left(\left|C_{1}\right|,\left|C_{2}\right|, \ldots,\left|C_{k}\right|\right)
$$

and say that $\pi$ is reducible if $\operatorname{gcd}(\pi) \geq 2$.

With this definition, we have the following result.

Theorem 4 Let $G$ be a weighted undirected graph and suppose that $\pi=\left\{C_{1}, C_{2}, \ldots, C_{k}\right\}$ is an almost equitable partition of $G$, where $k \geq 2$. Suppose that $\pi$ is reducible and let

$$
q_{j}:=\frac{\left|C_{j}\right|}{\operatorname{gcd}(\pi)}
$$

for $j=1,2, \ldots, k$. Let $d$ be an integer such that $1 \leq d \leq$ $\operatorname{gcd}(\pi)-1$ and let $\mathbf{b} \in\{0,1\}^{n}$ be such that

$$
\left|\chi(\mathbf{b}) \cap C_{j}\right|=d q_{j}
$$

for all $j=1,2, \ldots, k$. Then $(\mathbf{L}, \mathbf{b})$ is uncontrollable. In particular, there are $\sum_{d=1}^{\operatorname{gcd}(\pi)-1} \prod_{j=1}^{k}\left(\begin{array}{c}\left|C_{j}\right| \\ d q_{j}\end{array}\right)$ such choices for $\mathbf{b}$.

PROOF. Since $\pi$ is reducible, $\operatorname{gcd}(\pi) \geq 2$ and therefore $q_{j} \geq 1$ is an integer and $\operatorname{gcd}(\pi)-1 \geq 1$. Since $d<\operatorname{gcd}(\pi)$, we have $d q_{j}<\left|C_{j}\right|$ for all $j=1,2, \ldots, k$. Therefore, if $\mathbf{b}$ is chosen to satisfy (2) then $\mathbf{b}$ is not constant on the cells of $\pi$ (that is, $\mathbf{b} \notin \operatorname{img}(\mathbf{P}(\pi))$ ) and thus we cannot deduce uncontrollability of $(\mathbf{L}, \mathbf{b})$ from Theorem 3 . Now, since $\pi$ is a non-trivial AEP, there exists an eigenvector $\mathbf{w} \in \operatorname{img}(\mathbf{P}(\pi))$ of $\mathbf{L}$ such that $\mathbf{w} \neq \mathbf{1}_{n}$. Let $\mathbf{c}_{j}$ be the characteristic vector of the cell $C_{j}$ for $j=1,2, \ldots, k$. Therefore, since $\left\{\mathbf{c}_{1}, \ldots, \mathbf{c}_{k}\right\}$ is a basis for $\operatorname{img}(\mathbf{P}(\pi))$, there exists $\alpha_{1}, \ldots, \alpha_{k} \in \mathbb{R}$ such that $\mathbf{w}=\sum_{j=1}^{k} \alpha_{j} \mathbf{c}_{j}$. Since both $\mathbf{w}$ and $\mathbf{1}_{n}$ are eigenvectors of the symmetric matrix $\mathbf{L}$, it follows that $0=\mathbf{w}^{T} \mathbf{1}_{n}=\sum_{j=1}^{k} \alpha_{j}\left|C_{j}\right|$. Hence, if $\mathbf{b}$ satisfies (2) then

$$
\begin{aligned}
\mathbf{w}^{T} \mathbf{b}=\sum_{j=1}^{k} \alpha_{j}\left|\chi(\mathbf{b}) \cap C_{j}\right| & =\sum_{j=1}^{k} \alpha_{j} d q_{j} \\
& =\sum_{j=1}^{k} \alpha_{j} d \frac{\left|C_{j}\right|}{\operatorname{gcd}(\pi)} \\
& =\frac{d}{\operatorname{gcd}(\pi)} \sum_{j=1}^{k} \alpha_{j}\left|C_{j}\right|=0 .
\end{aligned}
$$

Since $\mathbf{L}$ is symmetric, it follows by the $\mathrm{PBH}$ test that $(\mathbf{L}, \mathbf{b})$ is uncontrollable.

Let $d$ be fixed. For each fixed $j \in\{1,2, \ldots, k\}$, the number of ways to choose $d q_{j}$ of the indices of $\mathbf{b}$ such that $\left|\chi(\mathbf{b}) \cap C_{j}\right|=d q_{j}$ is $\left(\begin{array}{c}\left|C_{j}\right| \\ d q_{j}\end{array}\right)$. Now, since $C_{1}, \ldots, C_{k}$ are mutually disjoint, the number of leader selections b such that $\left|\chi(\mathbf{b}) \cap C_{j}\right|=d q_{j}$ for all $j=1,2, \ldots, k$ is $\prod_{j=1}^{k}\left(\begin{array}{c}\left|C_{j}\right| \\ d q_{j}\end{array}\right)$. If we now add up all such leader selections for each $d$ we clearly obtain $\sum_{d=1}^{\operatorname{gcd}(\pi)-1} \prod_{j=1}^{k}\left(\begin{array}{c}\left|C_{j}\right| \\ d q_{j}\end{array}\right)$.

We further illustrate the use of Theorem 4 on the asymmetric 3-regular Frucht graph [9], which as in Example 3, can be used to account for a substantial number of the uncontrollable leader selections.

Example 4 Consider the asymmetric 3-regular Frucht graph $G$ on $n=12$ vertices shown in Figure 5 . Since $G$ is a regular graph, almost equitable partitions are automatically equitable partitions. We compute that the only non-trivial equitable partitions of $G$ are

$$
\begin{aligned}
& \pi_{1}=\{\{3,7,10\},\{1,2,4,5,6,8,9,11,12\}\} \\
& \pi_{2}=\{\{1,5,7,12\},\{3,6,9,11\},\{2,4,8,10\}\} \\
& \pi_{3}=\{\{1,5,7,12\},\{2,3,4,6,8,9,10,11\}\} .
\end{aligned}
$$

Consider first the partition $\pi_{1}=\left\{C_{1}, C_{2}\right\}$, which has $\operatorname{gcd}\left(\pi_{1}\right)=3$, and thus $q_{1}=1$ and $q_{2}=3$. Applying Theorem 4 , if $\mathbf{b}$ is such that $\left|\chi(\mathbf{b}) \cap C_{1}\right|=d$ and $\mid \chi(\mathbf{b}) \cap$ $C_{2} \mid=3 d$, where $d \in\{1,2\}$, then $(\mathbf{L}, \mathbf{b})$ is uncontrollable. There are $\left(\begin{array}{l}3 \\ 1\end{array}\right)\left(\begin{array}{l}9 \\ 3\end{array}\right)+\left(\begin{array}{l}3 \\ 2\end{array}\right)\left(\begin{array}{l}9 \\ 6\end{array}\right)=504$ such choices for $\mathbf{b}$.

Consider now the partition $\pi_{2}=\left\{C_{1}, C_{2}, C_{3}\right\}$, which has $\operatorname{gcd}\left(\pi_{2}\right)=4$, and thus $q_{1}=q_{2}=q_{3}=1$. Applying Theorem 4 , if $\mathbf{b}$ is such that $\left|\chi(\mathbf{b}) \cap C_{j}\right|=d$ for $j=1,2,3$, where $d \in\{1,2,3\}$, then $(\mathbf{L}, \mathbf{b})$ is uncontrollable. There are $\left(\begin{array}{l}4 \\ 1\end{array}\right)^{3}+\left(\begin{array}{l}4 \\ 2\end{array}\right)^{3}+\left(\begin{array}{l}4 \\ 3\end{array}\right)^{3}=344$ such choices for $\mathbf{b}$.

Lastly, consider $\pi_{3}=\left\{C_{1}, C_{2}\right\}$, which has $\operatorname{gcd}\left(\pi_{3}\right)=4$, and thus $q_{1}=1$ and $q_{2}=2$. Applying Theorem 4 , if $\mathbf{b}$ is 
such that $\left|\chi(\mathbf{b}) \cap C_{1}\right|=d$ and $\left|\chi(\mathbf{b}) \cap C_{2}\right|=2 d$, where $d \in$ $\{1,2,3\}$, then $(\mathbf{L}, \mathbf{b})$ is uncontrollable. However, since $\pi_{2}$ is a refinement of $\pi_{3}$, we must not double count some b's already accounted for from $\pi_{2}$. Not including the ones that have already been accounted for from $\pi_{2}$, for $d=1$ there are $\left(\begin{array}{l}4 \\ 1\end{array}\right)\left[2\left(\begin{array}{l}4 \\ 2\end{array}\right)\right]=48$ choices for $\mathbf{b}$, for $d=2$ there are $\left(\begin{array}{l}4 \\ 2\end{array}\right)\left[2\left(\begin{array}{l}4 \\ 0\end{array}\right)\left(\begin{array}{l}4 \\ 4\end{array}\right)+2\left(\begin{array}{l}4 \\ 1\end{array}\right)\left(\begin{array}{l}4 \\ 3\end{array}\right)\right]=204$ choices for $\mathbf{b}$, and for $d=3$ there are $\left(\begin{array}{l}4 \\ 3\end{array}\right)\left[2\left(\begin{array}{l}4 \\ 4\end{array}\right)\left(\begin{array}{l}4 \\ 2\end{array}\right)\right]=48$ choices for b. Hence, there are 300 uncontrollable leader-selections corresponding to $\pi_{3}$.

In summary, with Theorem 4 we can account for 1148 uncontrollable leader selections. On the other hand, it can be shown that with Theorem 3 we can account for only 8 uncontrollable leader selections.

Remark 4 Let $G$ be a graph on $n$ vertices and let $\pi=\left\{C_{1}, C_{2}, \ldots, C_{k}\right\}$ be a partition of $V(G)$. Then $n=\sum_{j=1}^{k}\left|C_{j}\right|$ and therefore if $n$ is prime, necessarily $\operatorname{gcd}(\pi)=1$. Hence, in this case, $G$ contains no reducible AEPs, and thus Theorem 4 is not applicable. This suggests that networks with a prime number of nodes may have robust controllability properties.

Using quotient graphs, we give a further case in which the eigenvectors in $\operatorname{img}(\mathbf{P}(\pi))$ are orthogonal to leader selections not in $\operatorname{img}(\mathbf{P}(\pi))$. The situation arises when $\pi$, in contrast to Theorem 4 , is not necessarily reducible.

Theorem 5 Let $G$ be a weighted undirected graph and suppose that $\pi=\left\{C_{1}, C_{2}, \ldots, C_{k}\right\}$ is an almost equitable partition of $G$ and let $G / \pi$ be the induced quotient graph. Suppose that $\rho=\left\{S_{1}, S_{2}, \ldots, S_{\ell}\right\}$ is a non-trivial almost equitable partition of $G / \pi$. For each $i=1,2, \ldots, \ell$, enumerate the elements of $S_{i}$ as $S_{i}=\left\{C_{i, 1}, C_{i, 2}, \ldots, C_{i, k_{i}}\right\}$. Let $\mathbf{b} \in\{0,1\}^{n}$ and set $q_{i, j}:=\left|\chi(\mathbf{b}) \cap C_{i, j}\right|$. Assume that if $k_{i} \geq 2$ then $q_{i, j}$ is independent of $j \in\left\{1,2, \ldots, k_{i}\right\}$. Then $(\mathbf{L}, \mathbf{b})$ is uncontrollable.

PROOF. Let $\mathrm{W}_{1}=\operatorname{img}(\mathbf{P}(\pi))$ and we note that because $\rho$ is an almost equitable partition of $G / \pi$, $\operatorname{img}(\mathbf{P}(\rho))$ is $\left.\mathbf{L}\right|_{\mathbb{W}_{1}}$-invariant. Therefore, $\operatorname{ker}\left(\mathbf{P}(\rho)^{T}\right)$ is also $\left.\mathbf{L}\right|_{\mathrm{W}_{1}}$-invariant since $\left.\mathbf{L}\right|_{\mathrm{W}_{1}}$ is self-adjoint. Since $\rho$ is a non-trivial partition, there exists an eigenvector $\widetilde{\mathbf{w}} \in \operatorname{ker}\left(\mathbf{P}(\rho)^{T}\right)$ of $\mathbf{L}_{\pi}$ (note that $\mathbf{L}_{\pi}$ is a matrix representation of $\left.\mathbf{L} \mid \mathrm{W}_{1}\right)$. By Lemma $2, \mathbf{w}=\mathbf{P}(\pi) \widetilde{\mathbf{w}}$ is an eigenvector of $\mathbf{L}$. Now, since $\mathbf{w} \in \operatorname{img}(\mathbf{P}(\pi))$ and $\rho$ is a partition of $V(G \backslash \pi)=\pi$, we can write

$$
\mathbf{w}=\sum_{i=1}^{\ell}\left(\sum_{j=1}^{k_{i}} \alpha_{i, j} \mathbf{c}_{i, j}\right),
$$

where $\mathbf{c}_{i, j}$ is the characteristic vector of cell $C_{i, j} \in S_{i}$. Now, since $\widetilde{\mathbf{w}} \in \operatorname{ker}\left(\mathbf{P}(\rho)^{T}\right)$ it follows that the components of $\mathbf{w}=\mathbf{P}(\pi) \widetilde{\mathbf{w}}$ on the cells $C_{i, 1}, \ldots, C_{i, k_{i}}$ sum to zero, in other words, $\sum_{j=1}^{k_{i}} \alpha_{i, j}=0$, for each $i=$ $1,2, \ldots, \ell$. Now, in the case that $S_{i}=\left\{C_{i, 1}\right\}$ is a singleton cell, then necessarily $\alpha_{i, 1}=0$ and thus $\mathbf{w}^{T} \mathbf{b}$ is independent of the value of $\mathbf{b}$ on the vertices in the cell
$C_{i, 1}$. Now, by construction,

$$
\begin{aligned}
\mathbf{w}^{T} \mathbf{b} & =\sum_{i=1}^{\ell}\left(\sum_{j=1}^{k_{i}} \alpha_{i, j}\left|\chi(\mathbf{b}) \cap C_{i, j}\right|\right) \\
& =\sum_{\substack{i=1 \\
k_{i} \geq 2}}^{\ell}\left(\sum_{j=1}^{k_{i}} \alpha_{i, j} q_{i, j}\right)=\sum_{\substack{i=1 \\
k_{i} \geq 2}}^{\ell} q_{i, j}\left(\sum_{j=1}^{k_{i}} \alpha_{i, j}\right)=0,
\end{aligned}
$$

where the second last equality follows since $q_{i, j}$ is independent of $j$. Thus, by the PBH test, $(\mathbf{L}, \mathbf{b})$ is uncontrollable.

The next example shows how Theorem 5 can be used when Theorem 4 is inapplicable.

Example 5 Consider again the asymmetric 3-regular Frucht graph on $n=12$ vertices shown in Figure 5 and studied in Example 4. One can show that $\rho=$ $\left\{\left\{C_{1}\right\},\left\{C_{2}, C_{3}\right\}\right\}$ is an equitable partition of $\mathbf{L}_{\pi_{2}}$. Here $S_{1}=\left\{C_{1}\right\}$ and $S_{2}=\left\{C_{2}, C_{3}\right\}$. Notice that the partition $\pi_{2} / \rho$ is precisely $\pi_{3}$. Now, from Theorem 5 , if $\mathbf{b}$ is such that $\left|\chi(\mathbf{b}) \cap C_{2}\right|=\left|\chi(\mathbf{b}) \cap C_{3}\right|=2$, and $\left|\chi(\mathbf{b}) \cap C_{1}\right|$ is arbitrary, then $(\mathbf{L}, \mathbf{b})$ is uncontrollable. Such choices of b are not all characterized by Theorem 4 . Indeed, applying Theorem 4 to $\pi_{2}$ directly as in Example 4, we must have that $\left|\chi(\mathbf{b}) \cap C_{j}\right|=d$ for $j=1,2,3$ where $d \in\{1,2,3\}$. Hence, the case $\left|\chi(\mathbf{b}) \cap C_{1}\right|=2$ is the only one that has been accounted for by Theorem 4 , but the cases $\left|\chi(\mathbf{b}) \cap C_{1}\right| \in\{0,1,3,4\}$ induce new uncontrollable leader selections characterized by Theorem 5 . There are a total of $\left(\begin{array}{l}4 \\ 2\end{array}\right)\left(\begin{array}{l}4 \\ 2\end{array}\right)\left[\left(\begin{array}{l}4 \\ 0\end{array}\right)+\left(\begin{array}{l}4 \\ 1\end{array}\right)+\left(\begin{array}{l}4 \\ 3\end{array}\right)+\left(\begin{array}{l}4 \\ 4\end{array}\right)\right]=360$ such choices. Similarly, if $\mathbf{b}$ is such that $\left|\chi(\mathbf{b}) \cap C_{2}\right|=$ $\left|\chi(\mathbf{b}) \cap C_{3}\right|=3$, and $\left|\chi(\mathbf{b}) \cap C_{1}\right| \in\{0,1,2,4\}$ then $(\mathbf{L}, \mathbf{b})$ is uncontrollable.

\section{Conclusion}

In conclusion, we considered the controllability problem for multi-agent networked control systems. The main results of the paper are two new graph-theoretic necessary conditions for controllability involving almost equitable partitions (Theorem 4 and Theorem 5). We also generalized the known results on the role of graph symmetries and uncontrollability to weighted digraphs (Theorem 3) and multiple-leaders. Our results show that the internal structure of communities in a graph can induce obstructions to controllability that cannot be characterized by symmetry arguments alone.

\section{References}

[1] C.O. Aguilar and B. Gharesifard. Graph controllability classes for the Laplacian leader-follower dynamics. IEEE Transactions on Automatic Control, 60(6):1611-1623, 2015.

[2] C.O. Aguilar and B. Gharesifard. Laplacian controllability classes for threshold graphs. Linear Algebra and Its Applications, 471:575-586, 2015.

[3] D.M. Cardoso, C. Delorme, and P. Rama. Laplacian eigenvectors and eigenvalues and almost equitable partitions. European Journal of Combinatorics, 28(3):665-673, 2007. 
[4] A. Chapman and M. Mesbahi. On symmetry and controllability of multi-agent systems. In 2014 IEEE 53rd Conf. on Decision and Control, pages 625-630, 2014.

[5] A. Chapman, M. Nabi-Abdolyousefi, and M. Mesbahi. Controllability and observability of network-of-networks via Cartesian products. IEEE Transactions on Automatic Control, 59(10):2668-2679, Oct 2014.

[6] G. Dullerud and F. Paganini. Course in Robust Control Theory. Springer-Verlag, New York, 2000.

[7] D.S. Dummit and R.M. Foote. Abstract Algebra, volume 1999. Prentice Hall Englewood Cliffs, NJ, 1991.

[8] P. Erdös and A. Réyni. Asymmetric graphs. Acta Math. Acad. Sci. Hungary, 14:295-315, 1963.

[9] R. Frucht. Herstellung von graphen mit vorgegebener abstrakter gruppe. Compositio Mathematica, 6:239-250, 1939.

[10] C. Godsil. Controllable subsets in graphs. Annals of Combinatorics, 16:733-744, 2012.

[11] C. Godsil and G. Royle. Algebraic Graph Theory. Springer, 2001.

[12] M. Golubitsky, I. Stewart, and A. Török. Patterns of synchrony in coupled cell networks with multiple arrows. SIAM Journal on Applied Dynamical Systems, 4(1):78-100, 2005.

[13] Z. Ji, H. Lin, and H. Yu. Leaders in multi-agent controllability under consensus algorithm and tree topology. Systems \& Control Letters, 61:918-925, 2012.

[14] M.-G.Yoon, P. Rowlinson D. Cvetković, and Z. Stanić. Controllability of multi-agent dynamical systems with a broadcasting control signal. Asian Journal of Control, 16(4):1066-1072, 2014.

[15] B.D. MacArthur, R. Sánchez-García, and J.W. Anderson. Symmetry in complex networks. Discrete Applied Mathematics, 156(18):3525-3531, 2008.

[16] S. Martini, M. Egerstedt, and A. Bicchi. Controllability analysis of multi-agent systems using relaxed equitable partitions. International Journal of Systems, Control and Communications, 2(1):100-121, 2010.

[17] B. McKay and A. Piperno. Practical graph isomorphism, II. Journal of Symbolic Computation, 60:94-112, 2013.

[18] N. Monshizadeh, H.L. Trentelman, and M.K. Camlibel. Projection-based model reduction of multi-agent systems using graph partitions. IEEE Transactions on Control of Network Systems, 1(2):145-154, June 2014.

[19] N. Monshizadeh, S. Zhang, and M.K. Camlibel. Zero forcing sets and controllability of dynamical systems defined on graphs. IEEE Transactions on Automatic Control, 59(9):2562-2567, 2014.

[20] N. Monshizadeh, S. Zhang, and M.K. Camlibel. Disturbance decoupling problem for multi-agent systems: A graph topological approach. Systems \& Control Letters, 76:35-41, 2015.

[21] M. Nabi-Abdolyousefi and M. Mesbahi. On the controllability properties of circulant networks. IEEE Transactions on Automatic Control, 58(12):3179-3184, December 2013.

[22] G. Notarstefano and G. Parlangeli. Controllability and observability of grid graphs via reduction of symmetries. IEEE Transactions on Automatic Control, 58(7):1719-1731, 2013.

[23] G. Parlangeli and G. Notarstefano. On the reachability and observability of path and cycle graphs. IEEE Transactions on Automatic Control, 57(3):743-748, 2012.
[24] A. Rahmani, M. Ji, M. Mesbahi, and M. Egerstedt. Controllability of multi-agent systems from a graph-theoretic perspective. SIAM Journal on Control and Optimization, 48(1):162-186, 2009.

[25] I. Stewart, M. Golubitsky, and M. Pivato. Symmetry groupoids and patterns of synchrony in coupled cell networks. SIAM Journal on Applied Dynamical Systems, 2(4):609-646, 2003.

[26] H.G. Tanner. On the controllability of nearest neighbor interconnections. In IEEE Conf. on Decision and Control, pages 2467-2472, 2004.

[27] T. Tao and V. Vu. Random matrices have simple spectrum. Combinatorica, 2016. to appear.

[28] S. Zhang, M. Cao, and M.K. Camlibel. Upper and lower bounds for controllable subspaces of networks of diffusively coupled agents. IEEE Transactions on Automatic Control, 59(3):745-750, March 2014.

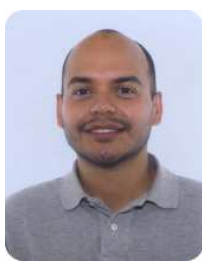

Cesar O. Aguilar received the B.Sc. degree in Mathematics and Engineering in 2003 from Queen's University (Canada), and the M.Sc. degree in Electrical and Computer Engineering in 2005 from the University of Alberta. He received the Ph.D. degree in Mathematics from Queen's University in 2010. He held a National Research Council postdoctoral fellowship in the Department of Applied Mathematics at the Naval Postgraduate School in Monterey, CA from 2010-2013. From 2013-2016, he was an Assistant Professor with the Department of Mathematics at California State University, Bakersfield. He is currently an Assistant Professor with the Department of Mathematics at the State University of New York at Geneseo. His research interests include nonlinear controllability theory, output regulation, optimal control, networked dynamical systems, and numerical methods in control theory.

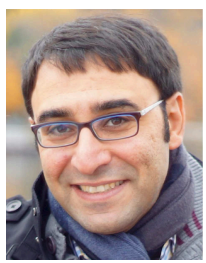

Bahman Gharesifard received the B.Sc. degree in Mechanical Engineering, in 2002, and the M.Sc. degree in Control and Dynamics, in 2005, from Shiraz University, Iran. He received the Ph.D. degree in Mathematics, in 2009, from Queen's University, Canada. He held postdoctoral positions with the Department of Mechanical and Aerospace Engineering at University of California, San Diego 2009-2012 and with the Coordinated Science Laboratory at the University of Illinois at Urbana-Champaign from 2012-2013. He held a visiting faculty position at the Institute for Systems Theory and Automatic Control at the University of Stuttgart in summer of 2016. He is currently an Assistant Professor with the Department of Mathematics and Statistics at Queen's University. His research interests include systems and controls, distributed control and optimization, social and economic networks, game theory, geometric control and mechanics, and Riemannian geometry. 